\title{
TRANSSPHENOIDAL SURGERY, GAMMA-KNIFE SURGERY AND DIABETES ARE THE FACTORS AFFECTING THE QUALITY OF LIFE IN ACROMEGALIC PATIENTS
}

\author{
Ayse Serap Yalin, Seda Sancak, Oguzhan Deyneli, Sule Temizkan, Mutlu Gunes, Dilek Yavuz, N.Sema Akalin \\ Marmara University Medical School, Section of Endocrinology and Metabolism Istanbul, Turkey
}

\begin{abstract}
AIM: Acromegaly is a chronic disease with an important impact on patients, Health Related Quality of Life (HRQoL). Acromegaly Quality of Life Questionnaire (AcroQoL) is a disease-generated QoL questionnaire comprising 22 questions each having five possible responses scored 1-5, the maximum score of 110 reflecting best possible QOL, and quoted as a percentage. The 22 items break down into two categories, physical and psychological function, the latter being subdivided into appearance and personal relationships. We aimed to investigate the factors affecting the QoL in acromegalic patients by using AcroQoL.
\end{abstract}

MATERIAL-METHODS: Sixty- five acromegalic patients ( 37 male, 28 female) were enrolled in this cross-sectional study. Patients completed AcroQoL. Patients medical history and laboratory findings were available from patients past medical records.

Table-1: Characateristics of acromegalic patients

\begin{tabular}{|l|c|}
\hline & $\begin{array}{l}\text { Acromegalic } \\
\text { patients (n=65) }\end{array}$ \\
\hline Diabetes Mellitus (\%) & $18(27.7)$ \\
\hline Hypertension (\%) & $28(43.1)$ \\
\hline Hyperlipidemia (\%) & $20(32.3)$ \\
\hline Hypopituitarism (\%) & $19(29.2)$ \\
\hline $\begin{array}{l}\text { Transsphenoidal surgery } \\
\text { (TSS) (\%) }\end{array}$ & $55(84.6)$ \\
\hline $\begin{array}{l}\text { Gammaknife } \\
\text { radiosurgery (GKS) (\%) }\end{array}$ & $39(60)$ \\
\hline $\begin{array}{l}\text { Somatostatain analog } \\
\text { (SA) (\%) }\end{array}$ & $41(63.1)$ \\
\hline $\begin{array}{l}\text { Patients with normal } \\
\text { IGF-1 (uncontrolled (\%) }\end{array}$ & $24(36.9)$ \\
\hline $\begin{array}{l}\text { Patients with suppressed } \\
\text { GH (\%) }\end{array}$ & $20(30.8)$ \\
\hline
\end{tabular}

RESULTS: Age (mean \pm SD: $44.75 \pm 10.81$ year), body mass index (mean \pm SD: $29.77 \pm 4.20 \mathrm{~kg} / \mathrm{m} 2)$, disease duration (median [IQR]: 48 [12-103] months), basal growth hormone $(\mathrm{GH})$ (median [IQR]: 1,66 [0.69-6.05] ng/dL), nadir GH (median [IQR]: 1,13 [0.47-4.80] ng/dL) and insulin like growth factor (IGF-1) (median [IQR]: 311 [199-504.5] ng/dL) were evaluated. Characteristics of acromegalic patients were summarized in Table-1. Disease control was evaluated by IGF-1 (referance values per age) and $\mathrm{GH}$ levels (random $\mathrm{GH}<1 \mathrm{ng} / \mathrm{dL}$ in patients on somatostatain analog $[\mathrm{SA}]$ and nadir $\mathrm{GH}<0.4 \mathrm{ng} / \mathrm{dL}$ in patients not on SA). Twenty-four (36.9\%) patients were under control according to IGF-1 criteria, while 20 (\%30.8) patients were under control according to $\mathrm{GH}$ criteria. No correlation was found between AcroQoL scores and any of the parameters. AcroQoL scores were lower in patients with diabetes (52 vs $67, \mathrm{p}<0.05)$ and who had radiotherapy ( 59 vs $75, \mathrm{p}<0.05$ ). However, patients who underwent transsphenoidal surgery (TSS) had higher scores ( 66 vs 39, $\mathrm{p}<0.05$ ).

Table-2: Factors affecting AcroQoL scores

\begin{tabular}{|l|c|c|c|}
\hline & $\begin{array}{l}\text { AcroQoL Scores } \\
\text { of patients with }\end{array}$ & $\begin{array}{l}\text { AcroQoL Scores of } \\
\text { patients without }\end{array}$ & p value \\
\hline Diabetes Mellitus & 52 & 67 & $<0.05$ \\
\hline $\begin{array}{l}\text { Transsphenoidal } \\
\text { surgery (TSS) }\end{array}$ & 66 & 39 & $<0.05$ \\
\hline $\begin{array}{l}\text { Gammaknife } \\
\text { radiosurgery (GKS) }\end{array}$ & 59 & 75 & $<0.05$ \\
\hline
\end{tabular}

CONCLUSIONS: Our results demonstrated that TSS, GKS and co-existing diabetes were the only factors affecting acromegaly related QoL. 